\title{
摩-磁复合制动性能及恒力矩制动控制研究
}

\author{
鲍久圣 ${ }^{1,2}$ 纪洋洋 ${ }^{1}$ 阴妍 ${ }^{1}$ 黄 山 ${ }^{1}$ \\ (1. 中国矿业大学机电工程学院 徐州 221116;
}

2. 中国矿业大学江苏省矿山智能采掘装备协同创新中心 徐州 221008)

\begin{abstract}
摘要: 摩-磁复合制动将摩擦制动和磁力制动的优点有机结合起来, 是一种很有前途的新型制动方式, 但目前对摩-磁复合制 动性能及其调控方法的研究还存在很大不足。利用摩-磁复合制动性能检测试验台, 开展了有、无磁场作用下的模拟制动试验, 分析了摩-磁复合制动性能的变化规律; 提出制动过程恒力矩制动的控制目标, 利用 BP 神经网络和模糊 PID 等智能技术分别 设计建立了基于磁场的摩-磁复合制动性能智能调控方法; 分别开展了常规摩擦制动试验和恒力矩制动试验, 并对试验结果进 行了分析。结果表明, 通过输入制动压力和制动初速度两个参数, 所建立的 BP 神经网络预测模型可以有效地预测摩擦制动 力矩终值; 通过降低制动力矩的波动幅值, 所建立的模糊 PID 反馈调控法可以实现制动系统以近似恒力矩制动; 相比常规的 摩擦制动, 摩-磁复合制动方式拥有更大的制动力矩和更短的制动时间, 表现出更好的制动性能。
\end{abstract}

关键词: 摩-磁复合; 恒力矩制动; 制动性能; 神经网络; 模糊 PID

中图分类号: TH117; TP183

\section{Braking Performance and Constant Torque Controlling of Frictional-magnetic Compound Brake}

\author{
BAO Jiusheng $^{1,2}$ JI Yangyang ${ }^{1}$ YIN Yan ${ }^{1}$ HUANG Shan ${ }^{1}$ \\ (1. School of Mechanical and Electrical Engineering, China University of Mining and Technology, Xuzhou 221116; \\ 2. Jiangsu Collaborative Innovation Center of Intelligent Mining Equipment, \\ China University of Mining and Technology, Xuzhou 221008)
}

\begin{abstract}
As a new type of braking, the frictional-magnetic compound brake could combine the advantages of both frictional braking and magnetic braking, which is considered as an excellent braking method. However, for the frictional-magnetic compound brake's braking performance and controlling method, few researches are studied in the past. Firstly, based on a frictional-magnetic compound brake tester, the braking performance under and without magnetic field are carried out respectively. The changing rules of braking performance are analysed by experiments. Secondly, on the basis of the changing rule of braking performance, a control target of the constant force moment brake of the braking process is proposed. The intelligent control method for braking performance of frictional-magnetic compound brake is designed and established by using BP neural network and fuzzy PID and other intelligent techniques. At last, conventional friction braking tests and the constant force moment brake tests are carried out respectively. The results show that the final prediction model of friction braking torque based on BP neural network can effectively predict the final value of friction braking torque according to working parameters: initial braking speed and braking pressure. The feedback control method based on fuzzy PID can effectively reduce the fluctuating amplitude of braking torque. The braking system is implemented to braking with an approximate constant brake torque. In the braking process of frictional-magnetic compound brake, the braking speed is greater, the braking time is shorter, and the braking performance is further improved.
\end{abstract}

Key words: frictional-magnetic compound; constant brake torque; braking performance; neural network; fuzzy PID

\section{0 前言}

目前, 交通车辆和机械设备的制动方式根据原

\footnotetext{
* 国家自然科学基金(51875561)、摩擦学国家重点实验室开放基金 (SKLTKF13B09)和江苏省高校优势学科建设工程资助项目。20180304 收到初稿, 20180725 收到修改稿
}

理不同主要可分为摩擦制动和磁力制动两种。尽管 摩擦制动的制动效率和可靠性较高, 但由于其不可 避免的制动热衰减现象, 在极端制动工况下易发生 “摩擦突变”, 严重影响制动性能, 甚至引发制动失 效, 造成难以估量的损失 ${ }^{[1-3]}$ 。作为一种非接触的制 动方式, 磁力制动具有经济环保的优点, 但是其在 低速时制动效果差且无法实现可靠停止 ${ }^{[4-5]}$ 。因此结 
合摩擦制动与磁力制动优点的集成系统一一摩-磁 复合制动方式应运而生, 但现有研究提出的复合制 动器结构集成度较低, 所占空间大 ${ }^{[6-8]}$ 。

在控制策略方面, 现有研究主要是以多种制动 环境及工况为依据, 在磁力制动和摩擦制动之间进 行切换和组合, 以合理分配制动力矩、提高制动性 能; 例如: 在短时、低强度制动时采用磁力制动以 减少由摩擦制动带来的摩擦副寿命损耗, 而在需要 高强度制动或制动停止的条件下, 则使用两种制动 方式同时进行制动 ${ }^{[9-10]}$ 。在对两种制动方式的具体 控制方法上, 现有研究大都通过多种传感器来监测 制动过程的速度、温度和制动压力等参数的变化, 然后根据需要分别改变摩擦制动的油液压力和电磁 制动的电流大小, 进而实现对制动力矩的调节与分 配; 例如, 刘存香等 ${ }^{[11]}$ 设计了摩擦与电磁集成系统 结构与复合制动的控制策略, 通过多项对比试验验 证了所建立控制策略的正确性与可行性, 并通过抗 热衰退性试验证明了所设计的集成结构具有良好的 抗热衰退性; 何仁等 ${ }^{[12]}$ 提出了集成制动系统的切换 控制策略, 通过仿真分析认为该控制策略能够提高 整车制动性能, 同时给出了制动力矩的调整方法以 应对模式切换造成的力矩突变问题; 胡东海等 ${ }^{[13]}$ 设 计了摩擦与电磁集成制动系统解耦控制器, 提出 了集成系统制动模式切换控制策略, 建立集成制 动系统混杂特性模型并通过稳定性分析验证了模 型正确性。但是, 利用复合制动过程中制动性能 在不同工况下的变化规律来设计反馈调控方法,

现有研究还很少涉及。本文以摩-磁复合制动器在 磁场作用下的制动性能变化规律为理论基础, 利 用 BP 神经网络和模糊 PID 等智能技术设计并建立 摩-磁复合制动性能的反馈调控机制, 将恒力矩制 动作为控制目标进行试验和分析, 研究结果对于 提高机械设备制动系统的制动性能与可靠性、促 进多源复合制动系统调控技术的发展具有重要理 论价值和实际意义 ${ }^{[14]}$ 。

\section{1 试验设计}

\section{1 摩-磁复合制动性能检测试验装置}

采用自制摩-磁复合制动性能试验台模拟汽车 行驶过程中的惯量 ${ }^{[15]}$ 。摩-磁复合制动性能试验台主 要由摩-磁复合制动器、动力驱动系统、液压系统、 惯量载荷系统以及测控系统五个部分组成, 其整体 结构如图 1 所示。通过变频器控制电机调节试验台 转速, 通过液压系统实现摩擦制动, 通过向励磁线 圈通电流实现电磁制动, 电流、转速和转矩等参数
通过相应传感器测出, 参数的设置、信号的采集由 测控系统控制完成。

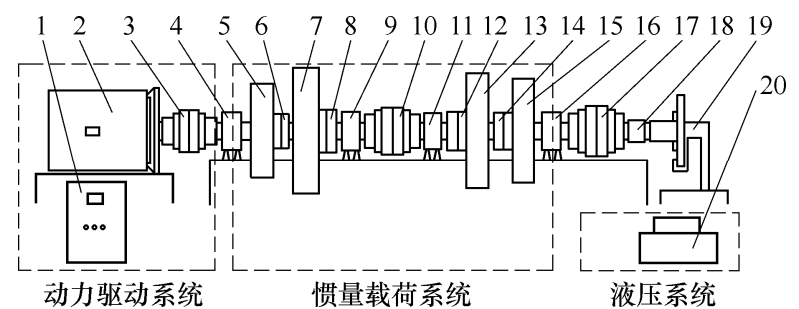

图 1 摩-磁复合制动性能试验台整体结构示意图 1. 变频控制器 2 . 调速电机 $3 、 10 、 17$. 联轴器

$4 、 9 、 11 、 16$. 轴承座 $5 、 7 、 13 、 15$, 惯性飞轮组 6、8、12、14. 电磁离合器 18 . 转速转矩传感器 19. 摩-磁复合制动器 20 . 液压站

\section{2 摩-磁复合盘式制动器样机}

采用自制摩-磁复合盘式制动器样机模拟制动 器工作的磁场环境以及实现磁力制动的功能 ${ }^{[16]}$ 。 摩-磁复合盘式制动器主要由制动盘、摩擦片、背板、 励磁线圈、钳体和钳架等组成, 其结构示意图如图 2 所示。每块背板均由两块钢板通过两个铁芯连接, 背板对称布置于制动盘两侧, 励磁线圈缠绕于铁芯 上, 通过向线圈中通入电流产生磁场。单个励磁线 圈的结构参数包括 ${ }^{[15]}$ : 内径 $D_{1}=24 \mathrm{~mm}$, 外径 $D_{2}=54$ $\mathrm{mm}$, 轴向宽度 $l=31 \mathrm{~mm}$, 㠴数 $N=438$, 铜导线直径 $d_{\mathrm{m}}=0.9 \mathrm{~mm}$ 。 4 个励磁线圈之间串联, 端电压最大为 $24 \mathrm{~V}$, 线圈中的电流最大值为 $8.14 \mathrm{~A}$ 。此外, 由于 普通摩擦片的磁导率很低, 因此采用本课题组发明 的、磁导率较高的摩擦片配方 ${ }^{[16]}$, 制备了导磁摩擦 片, 如图 3 所示。

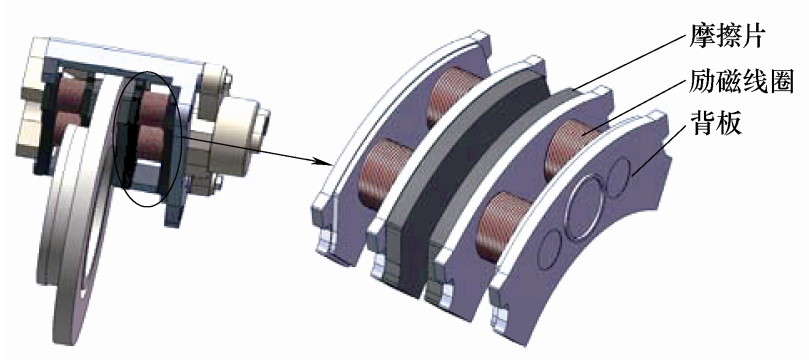

图 2 摩-磁复合盘式制动器样机结构示意图

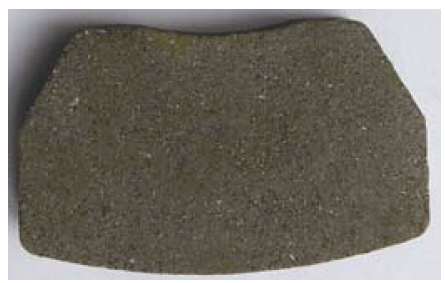

图 3 导磁摩擦片实物图

\section{3 试验方案}

在不同的工况条件下，使用单次制动的方法进 行摩-磁复合制动性能试验, 考察不同工况对摩-磁 复合制动性能的影响, 重要的工况参数主要包括磁 
感应强度、制动初速度和制动压力。励磁线圈的电 流大小与磁感应强度呈正相关关系, 根据线圈通入 电流的最大值 $8.14 \mathrm{~A}$, 试验通入电流的取值确定为 $1 \mathrm{~A} 、 2 \mathrm{~A} 、 3 \mathrm{~A} 、 4 \mathrm{~A} 、 5 \mathrm{~A} 、 6 \mathrm{~A} 、 7 \mathrm{~A} 、 8 \mathrm{~A}$; 根据汽 车在各种路况下的行车限速要求, 制动初速度的取 值确定为 $40 \mathrm{~km} / \mathrm{h} 、 60 \mathrm{~km} / \mathrm{h} 、 80 \mathrm{~km} / \mathrm{h} 、 100 \mathrm{~km} / \mathrm{h}$ 、 $120 \mathrm{~km} / \mathrm{h}$; 根据《轿车制动器台架试验方法 $\mathrm{QC} / \mathrm{T}$ 564-1999》行业标准及紧急制动工况, 拟定制动压 力的取值为 $0.8 \mathrm{MPa} 、 1.0 \mathrm{MPa} 、 1.2 \mathrm{MPa} 、 1.4 \mathrm{MPa}$ 、 $1.6 \mathrm{MPa}$ 。各试验参数的取值如表 1 所示。

表 1 摩-磁复合制动性能试验参数表

\begin{tabular}{lcrrccccc}
\hline \multicolumn{1}{c}{ 试验参数 } & \multicolumn{7}{c}{ 取值范围 } \\
\hline 电流 $/ \mathrm{A}$ & 1 & 2 & 3 & 4 & 5 & 6 & 7 & 8 \\
制动初速度 $/(\mathrm{km} / \mathrm{h})$ & 40 & 60 & 80 & 100 & 120 \\
制动压力 $/ \mathrm{MPa}$ & 0.8 & 1.0 & 1.2 & 1.4 & 1.6 \\
\hline
\end{tabular}

\section{2 摩-磁复合制动性能试验与分析}

制动力矩是反映摩-磁复合制动性能的重要参 数。由于复合制动过程中传感器无法分别检测到 电磁制动力矩和摩擦制动力矩的变化情况, 因此 本文首先在制动压力为零的条件下 (不进行摩擦制 动), 通过调节电流强度和制动盘转速进行单独的 电磁制动力矩检测试验, 然后拟合试验得到的数 据, 从而获得电流和转速与电磁制动力矩间的函 数关系。

\section{1 电磁制动力矩检测试验与分析}

(1) 电磁制动力矩检测试验。

本文对制动开始后 1 秒内的电磁制动力矩进行 采集, 将每个制动初速度对应的电磁制动力矩值近 似选为第 1 个采样点的值。按照上文试验参数的取 值范围, 在不同的电流和制动初速度下, 进行电磁 制动力矩检测试验, 使用 Matlab 软件根据试验数据 对电磁制动力矩与速度和电流之间的关系图进行拟 合, 如图 4 所示。

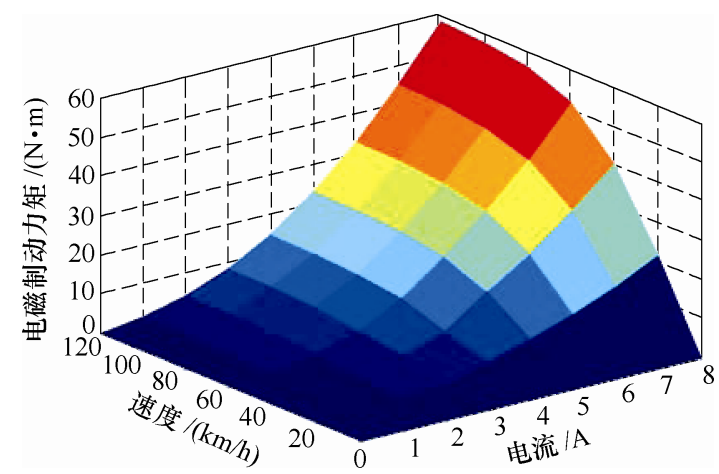

图 4 电磁制动力矩与速度和电流关系三维曲面图
由图 4 可知, 电磁制动力矩与制动初速度和电 流都成正相关关系，但电磁制动力矩随制动初速度 的增加上升得越来越慢, 而随着电流的增加上升得 越来越快。

（2）电磁制动力矩的函数表达式拟合。

由上文试验结果可以看出, 电磁制动力矩与电 流、制动初速度两个参数之间的关系是非线性变化 的, Matlab 中的 sftool 工具箱可以快速实现对多个 自变量计算整合，因此用其对上文获得的曲面图进 行电磁制动力矩与电流、制动初速度间的函数关系 式拟合, 得到公式(1)。

$$
\begin{gathered}
T_{d}=13.27+16.43 I+4.261 v+4.444 I^{2}+ \\
6.084 I v-3.327 v^{2}-0.006238 I^{3}+ \\
1.932 I^{2} v-3.239 I v^{2}+0.3896 v^{3}
\end{gathered}
$$

式中, $T_{d}$ 为电磁制动力矩; $I$ 为励磁电流; $v$ 为速度。

\section{2 无磁场作用下的制动性能检测试验}

在不向励磁线圈通电情况下，根据上文试验参 数分别开展相同制动压力、不同制动初速度以及相 同制动初速度、不同制动压力条件下的摩擦制动试 验, 得到摩擦制动力矩的变化情况分别如图 5、6 所示。限于篇幅, 仅给出部分代表性变化曲线进行 分析。在单次制动过程中, 摩擦制动力矩的初值和 终值分别由图中的 $A$ 点和 $B$ 点来表示。

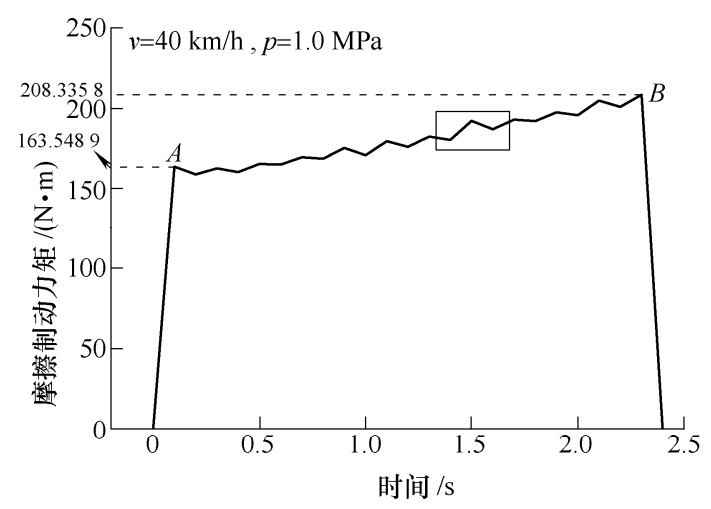

(a)

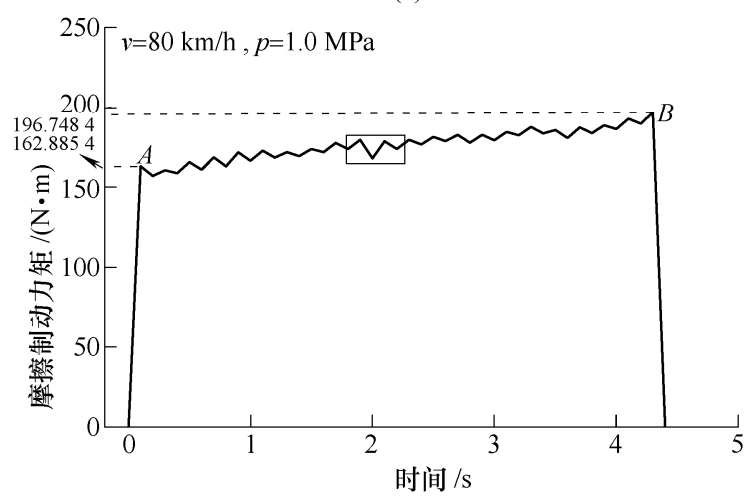

(b) 


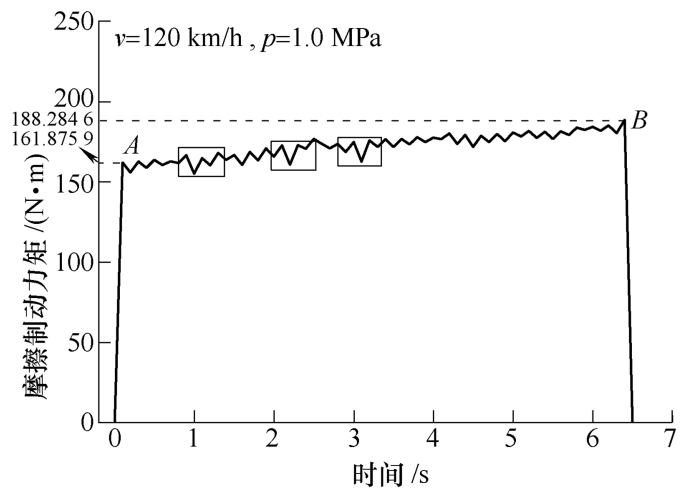

(c)

图 5 不同制动初速度下摩擦制动力矩随时间的变化曲线

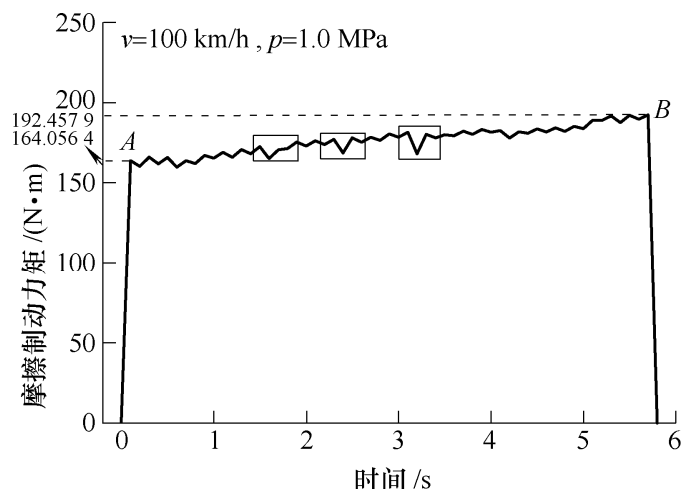

(a)

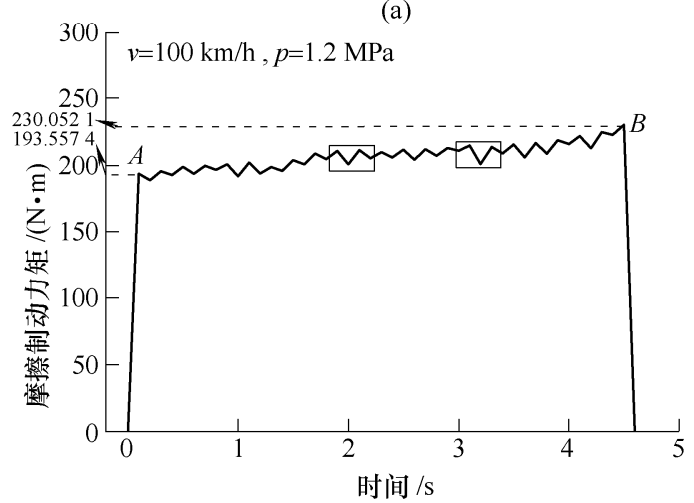

(b)

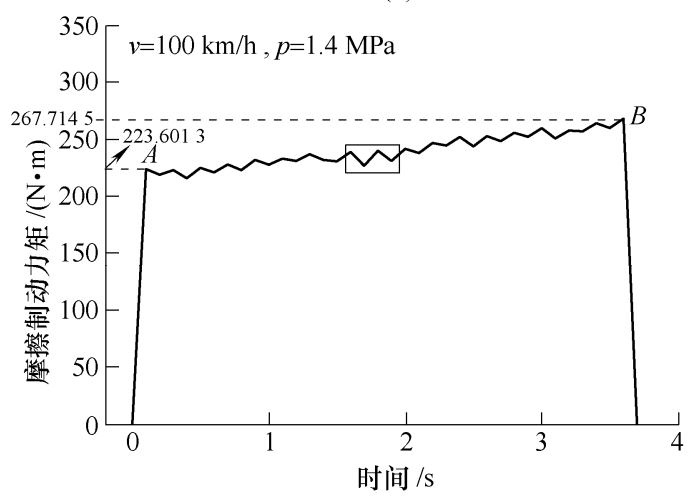

(c)

图 6 不同制动压力下摩擦制动力矩随时间的变化曲线

由图 5 可知, 在整个单次制动过程中, 摩擦制 动力矩整体变化趋势是动态上升的; 如图中方框标 注的位置, 有时会出现较为明显的波动现象; 摩擦 制动力矩增加的幅值 ( $B$ 与 $A$ 点纵坐标之差) 随着制
动初速度的增加而逐渐变小, 同时波动的数量和幅 值都逐渐变大。由此可以说明, 制动初速度的增大 使得摩擦制动力矩的变化更不稳定。

由图 6 可知, 在整个单次制动过程中, 摩擦制 动力矩增加的幅值 ( $B$ 与 $A$ 点纵坐标之差) 随制动压 力的增加而逐渐变大; 由图中方框标出位置可以看 到, 当制动压力的值较小时, 整个制动过程中存在 较多的摩擦制动力矩上下波动情况, 而这种明显的 波动变化情况随制动压力增加则变得越来越少。由 此可以说明, 制动压力的增大在一定程度上增加了 摩擦制动力矩变化的稳定性。

\section{3 磁场作用下的制动性能检测试验}

在磁场作用下进行两种制动方式共同作用的 摩-磁复合制动试验, 制动过程中的速度通过转速转 矩传感器测得, 把各速度代入式(1)计算得到相应电 磁制动力矩值, 再将此值与同时由转速转矩传感器 检测到的复合制动力矩(摩擦与电磁制动力矩之和) 作差, 即可算出摩擦制动力矩。

按照上文试验参数的取值范围开展摩-磁复合 制动性能检测试验, 选定以下参数为例进行分析: 制动初速度 $100 \mathrm{~km} / \mathrm{h}$ 、制动压力 $1.0 \mathrm{MPa}$, 电流 $1 \mathrm{~A}$ 至 $8 \mathrm{~A}$, 由此得到三种制动力矩(电磁制动力矩、摩 擦制动力矩和摩-磁复合制动力矩)在不同磁场强度 下随时间变化的曲线图(仅给出 4 组), 如图 7 所示。

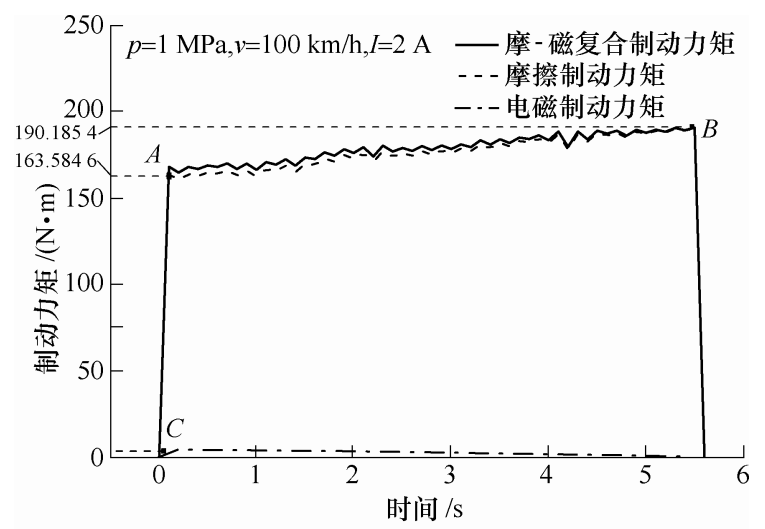

(a)

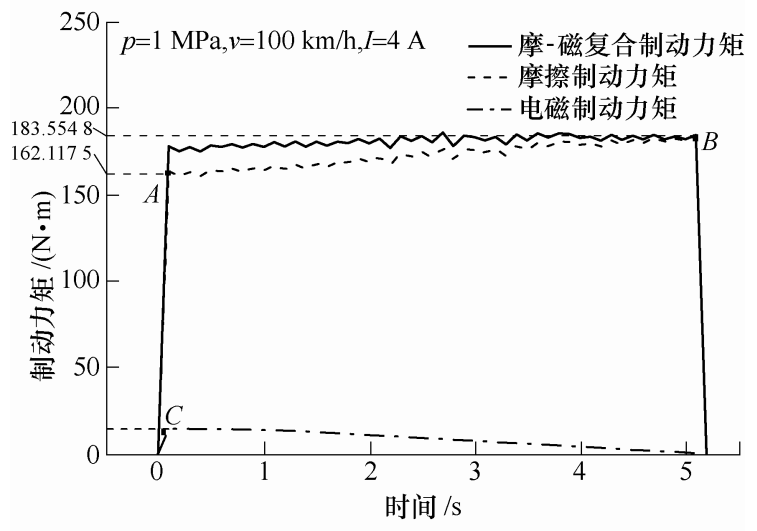

(b) 


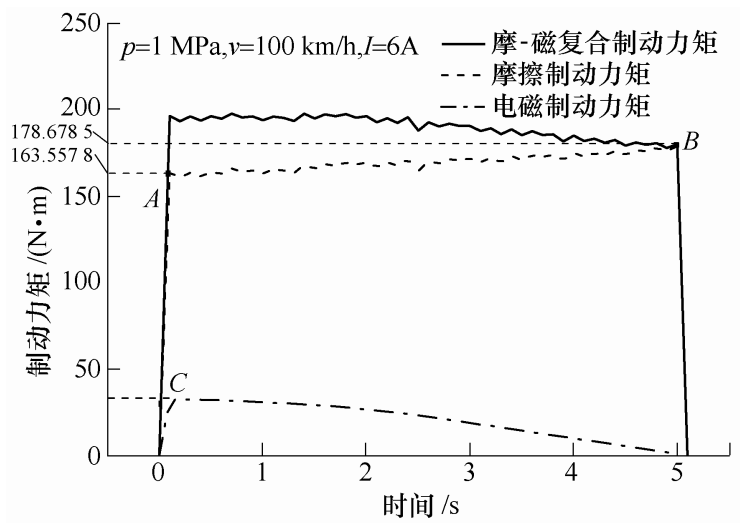

(c)

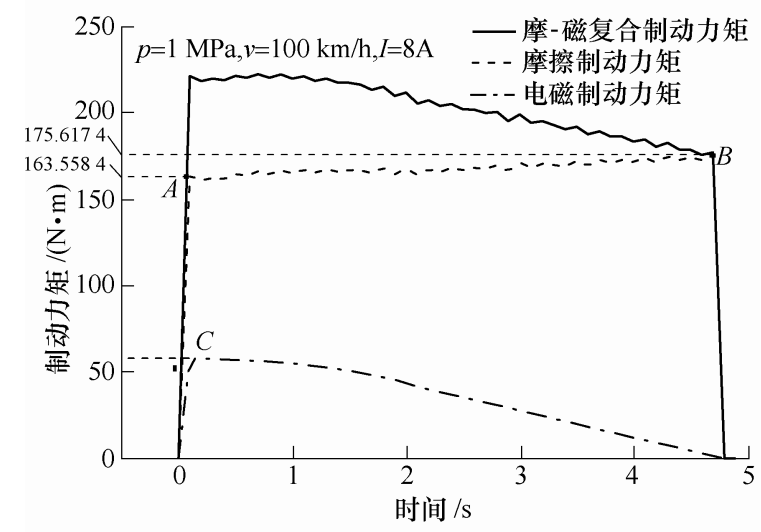

(d)

图 7 不同电流条件下制动力矩的变化曲线

由图 7 可以发现: (1) 在整个制动过程中, 电磁 制动力矩的值是逐渐变小的, 并且在开始降低的较 慢而后降低的速度逐渐增大; (2) 图中 $\mathrm{C}$ 点位置表 示电磁制动力矩的最大值, 对比几幅图可以看到, 整个制动过程中, 相应时段所对应的电磁制动力矩 值随电流值的增加而逐渐变大; (3) 摩-磁复合制动 力矩随电流值的增加也不断变大; 在制动结束时电 磁制动力矩降低为零, 此时摩擦制动力矩的值与摩磁复合制动力矩的值相等; (4) 与图 $6 \mathrm{a}$ 相比, 存在 磁场时的摩擦制动力矩增加的幅值 $(B$ 与 $A$ 点纵坐 标之差)较无磁场时有所降低, 并且增加的幅值随 电流值的增加而不断减小; (5) 在单次制动过程中 当摩擦制动力矩增加的幅值大于电磁制动力矩下 降的幅值时, 摩-磁复合制动力矩的值逐渐增加; 反之, 当摩擦制动力矩增加的幅值小于电磁制动力 矩下降的幅值时, 摩-磁复合制动力矩则表现出逐 渐下降的趋势。

\section{3 恒力矩调控方法研究}

\section{1 调控方法整体方案设计}

根据上文对摩-磁复合制动性能的研究可知, 制
动过程中, 制动力矩往往呈现不稳定的波动变化趋 势, 从而对制动器的制动性能及其稳定性造成影响。 为了建立响应快速和准确可靠的调控方法, 本文要 达到的目标有二：第一，以给定的制动压力和制动 初速度为依据, 对需要产生的电磁制动力矩的大小 进行快速决策和计算; 第二, 为减小制动力矩的波 动, 可以实时反馈制动力矩在制动过程中的变化情 况并进行相应调节。

智能调控摩-磁复合制动性能的原理如图 8 所 示。调控过程主要分为两大部分：1)在制动前通过 制动压力和初速度的输入, 系统进行快速判断决策, 计算出电磁制动力矩的初值; 2)在制动过程中通过 反馈机制不断对制动力矩进行调节, 以减小制动力 矩的波动变化情况, 以此实现近似恒力矩制动。判 断决策过程分为三步: 首先，由油液压力 $p$ 的大小 计算出摩擦制动力矩的理论初值 $T_{\mathrm{ms}}$; 其次, 神经 网络模型根据制动压力 $p$ 和制动初速度 $v$ 的输入对 摩擦制动力矩终值 $T_{\mathrm{me}}$ 进行预测; 最后, 将摩擦制 动力矩终值 $T_{\mathrm{me}}$ 和初值 $T_{\mathrm{ms}}$ 作差得到电磁制动力矩 $T_{d}$ 的值, 然后可以进一步计算出励磁电流 $I$ 的大小。 所建立的反馈调控机制具有如下作用：1)可以对制 动过程中的摩-磁复合制动力矩进行实时检测; 2)通 过改变励磁线圈中电流的大小来不断调节电磁制动 力矩的大小。

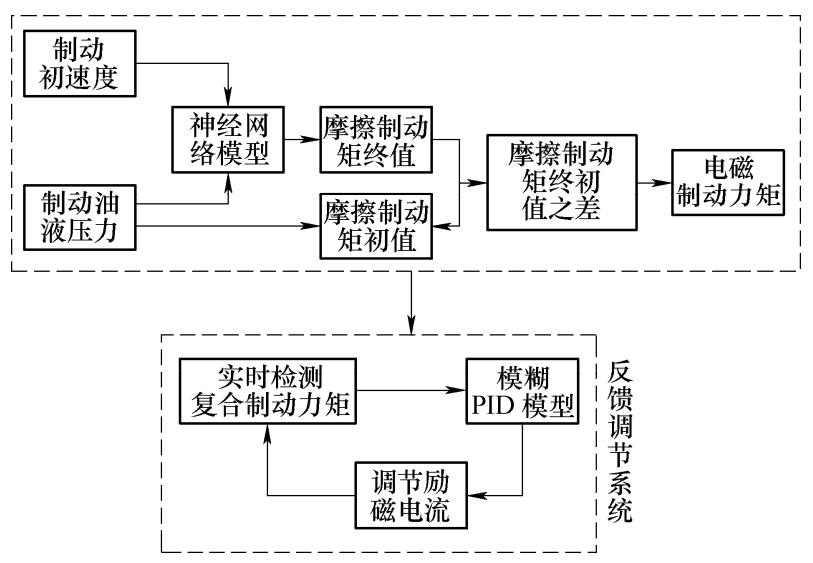

图 8 摩-磁复合制动性能智能调控过程原理图

\section{2 判断决策系统设计}

\subsection{1 摩擦制动力矩初值计算}

在不向励磁线圈中通电的情况下进行模拟制动 试验, 以制动初速度 $100 \mathrm{~km} / \mathrm{h}$ 为例, 按照上文试验 参数取值得到不同制动压力下的五组摩擦制动力矩 值。由于受摩擦系数的变化的影响, 摩擦制动力矩 在制动过程中呈现波动的状态, 因此本文将制动开 始时的制动力矩作为各个压力所对应的摩擦制动力 矩, 如图 9 所示。拟合试验曲线可看到油液压力与 摩擦制动力矩成正比, 计算出它们间的函数关系式 
如公式(2)。

$$
T_{\mathrm{ms}}=160.6783 p
$$

式中, $T_{\mathrm{ms}}$ 为摩擦制动力矩初值; $p$ 为制动压力。

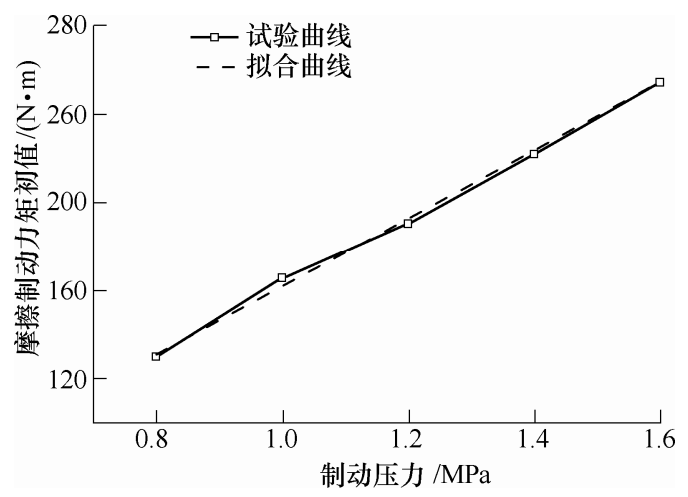

图 9 摩擦制动力矩初值随制动压力的变化曲线

\subsection{2 基于神经网络的摩擦制动力矩终值预测}

建立基于 BP 神经网络的摩擦制动力矩终值预 测模型, 首先需要对样本数据进行选择, 然后选定 模型的各结构参数。BP 神经网络预测模型主要由输 入层、隐含层和输出层组成, 其中输入层包括制动 压力和制动初速度, 输出层为摩擦制动力矩终值, 其结构示意图如图 10 所示。

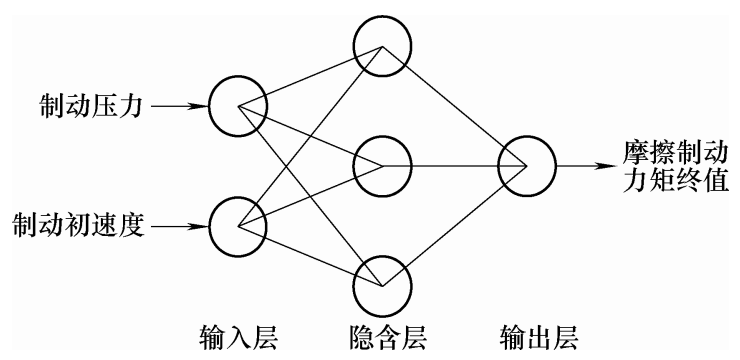

图 10 单个隐含层的 BP 神经网络模型结构示意图

(1) 样本数据选定。

根据试验参数的取值, 上文在无磁场作用时开 展的试验得到了 5 个不同制动初速度分别对应 5 个 不同制动压力下的制动性能检测结果, 共 25 组。为 了使 BP 神经网络模型的预测结果更为准确, 需要 得到更多的样本对其进行训练, 因此同样在上述 5 个制动压力下, 再补充制动初速度分别为 $50 \mathrm{~km} / \mathrm{h}$ 、 $70 \mathrm{~km} / \mathrm{h} 、 90 \mathrm{~km} / \mathrm{h}$ 和 $110 \mathrm{~km} / \mathrm{h}$ 时摩擦制动力矩检测 试验。最终得到样本数据共计 45 组, 随机选择 40 组作为训练样本, 剩余 5 组作为检验样本, 并对所 有样本数据进行归一化处理。

(2) 神经网络结构参数确定。

对 BP 神经网络结构的设计主要包括确定隐含 层层数及节点数、选择合适的传递函数、确定初始 权值和阈值、确定学习率以及选定训练函数, 具体 的参数值如表 2 所示。
表 2 BP 神经网络模型结构参数表

\begin{tabular}{lc}
\hline \multicolumn{1}{c}{ 参数名称 } & 参数值 \\
\hline 网络结构 & 3 层结构 $(1$ 输入层、 1 隐含层、 1 输出层 $)$ \\
输入层节点数 & 2 \\
隐含层节点数 & 4 \\
输出层节点数 & 1 \\
隐含层传递函数 & Tansig () \\
输出层传递函数 & Tansig () \\
训练函数 & Trainlm () \\
学习函数 & Learngdm () \\
学习率 & 0.01 \\
训练误差 & $<0.001$ \\
\hline
\end{tabular}

\section{3 基于模糊 PID 的闭环反馈调控系统}

\subsection{1 模糊 PID 控制器整体结构设计}

摩-磁复合制动性能的模糊 PID 控制器主要由 以下四部分组成: PID 控制器、模糊控制器、检测 装置和执行机构。其结构原理如图 11 所示。

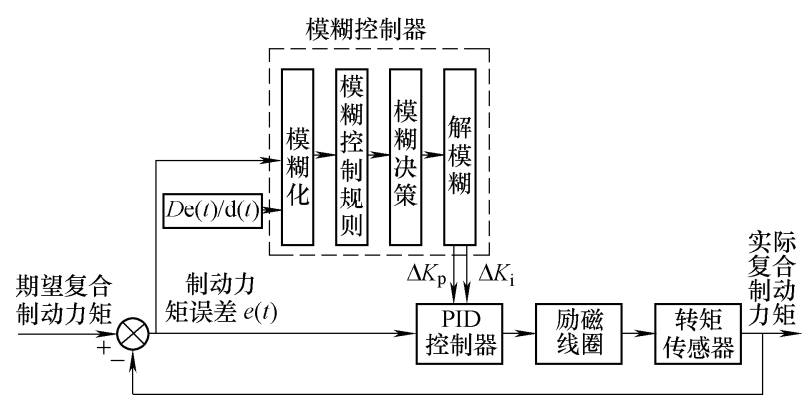

图 11 摩-磁复合制动性能的模糊 PID 控制器结构原理图

模糊 PID 控制器的输入量是期望制动力矩, 它 是通过 $\mathrm{BP}$ 神经网络预测得到的摩擦制动力矩终值。 模糊 PID 控制器的整体调控过程如下：1)首先比较 输入的期望制动力矩与制动时传感器检测到的实际 制动力矩, 计算出力矩的误差和变化率; 2)将该误 差数据传输到模糊控制器, 经模糊处理之后输出 PID 参数调整量; 3)将制动力矩误差和 PID 参数的 调整量共同传输到 PID 控制器中, PID 控制器处理 后获得调整之后的励磁线圈电流值; 4)电流值的改 变引起了实际制动力矩的变化, 调整后的制动力矩 反馈到之前的比较单元, 以此进行以上流程的循环。

\subsubsection{PID 控制器设计}

根据以上分析, 式(3)即反映了本文 PID 控制器 的作用: 即构建电流和制动力矩误差间的控制规则。

$$
u(t)=K_{\mathrm{p}}\left[e(t)+\frac{1}{T_{\mathrm{i}}} \int_{0}^{t} e(t) \mathrm{d} t+T_{\mathrm{d}} \frac{\mathrm{d} e(t)}{\mathrm{d} t}\right]
$$

式中, $T_{\mathrm{i}}$ 为积分时间常数; $T_{\mathrm{d}}$ 为微分时间常数; $K_{\mathrm{p}}$ 为比例系数; $K_{\mathrm{i}}$ 为积分系数, $K_{\mathrm{i}}=K_{\mathrm{p}} / T_{\mathrm{i}} ; K_{\mathrm{d}}$ 为微分 系数, $K_{\mathrm{d}}=K_{\mathrm{p}} \cdot T_{\mathrm{d}}$ 。

PID 控制器的调控环节主要包括比例、微分和 
积分。一般微分控制在时间滞后系统中的应用比较 常见, 为降低本文 PID 控制器的复杂性, “电流-力 矩” 系统的调控只使用比例和积分环节。最终通过 PLC 多次试凑, 确定 PID 调控效果最佳参数为: 积 分时间 $6 \mathrm{~s}$, 比例系数取 1 。

\subsection{3 模糊控制器设计}

模糊控制器的设计主要由三部分组成：模糊化 处理输入和输出变量、确定隶属函数和建立模糊控 制规则。

(1) 输入、输出变量模糊化。

首先需要模糊化处理输入和输出变量, 对它们 所对应的模糊集论域进行确定，同时使用模糊语言 表达它们。通过上文在不同工况下的制动性能检测 试验结果可以确定制动力矩的误差范围为 $-30 \sim 30$ $\mathrm{N} \cdot \mathrm{m}$, 而误差变化量的范围为 $-10 \sim 10 \mathrm{~N} \cdot \mathrm{m}$, 根据采 样频率 $10 \mathrm{~Hz}$, 误差变化率的范围为 $-100 \sim$ $100 \mathrm{~N} \cdot \mathrm{m} / \mathrm{s}$ 。由于制动时间较短, 因此将积分调整量 的基本论域定为 $[-0.3,0.3]$, 比例调整量的基本论域 定为 $[-0.6,0.6]$ 。最终 PID 参数调整量、制动力矩的 误差和误差变化率所对应的模糊集论域均确定为 $[-6,6]$, 模糊语言词集均为 $\{\mathrm{NB}, \mathrm{NM}, \mathrm{NS}, \mathrm{ZO}$, PS, PM, PB , 如表 3 所示。

表 3 输入、输出量模糊化处理结果

\begin{tabular}{cccccc}
\hline \multicolumn{3}{c}{ 名称 } & $\begin{array}{c}\text { 基本 } \\
\text { 论域 }\end{array}$ & $\begin{array}{c}\text { 模糊集 } \\
\text { 论域 }\end{array}$ & 模糊语言词集 \\
\hline \multirow{2}{*}{ 输入 } & $e$ & {$[-30,30]$} & {$[-6,6]$} & $\mathrm{NB}, \mathrm{NM}, \mathrm{NS}, \mathrm{ZO}, \mathrm{PS}, \mathrm{PM}, \mathrm{PB}$ \\
& $e_{\mathrm{c}}$ & {$[-100,100]$} & {$[-6,6]$} & $\mathrm{NB}, \mathrm{NM}, \mathrm{NS}, \mathrm{ZO}, \mathrm{PS}, \mathrm{PM}, \mathrm{PB}$ \\
& $\Delta K_{\mathrm{p}}$ & {$[-0.6,0.6]$} & {$[-6,6]$} & $\mathrm{NB}, \mathrm{NM}, \mathrm{NS}, \mathrm{ZO}, \mathrm{PS}, \mathrm{PM}, \mathrm{PB}$ \\
输出 & $\Delta K_{\mathrm{i}}$ & {$[-0.3,0.3]$} & {$[-6,6]$} & $\mathrm{NB}, \mathrm{NM}, \mathrm{NS}, \mathrm{ZO}, \mathrm{PS}, \mathrm{PM}, \mathrm{PB}$ \\
\hline
\end{tabular}

(2) 隶属函数确定。

为快速简单地获得线性分析结果，同时降低控 制系统的复杂性, 本文的输入、输出量的隶属函数 使用式(4)所示的三角型函数, 其隶属度的分布图如 图 12 所示。

$$
y=\left\{\begin{array}{lc}
0 & x<a \\
x-a / b-a & a \leqslant x<b \\
c-x / c-b & b \leqslant x \leqslant c \\
0 & x>c
\end{array}\right.
$$

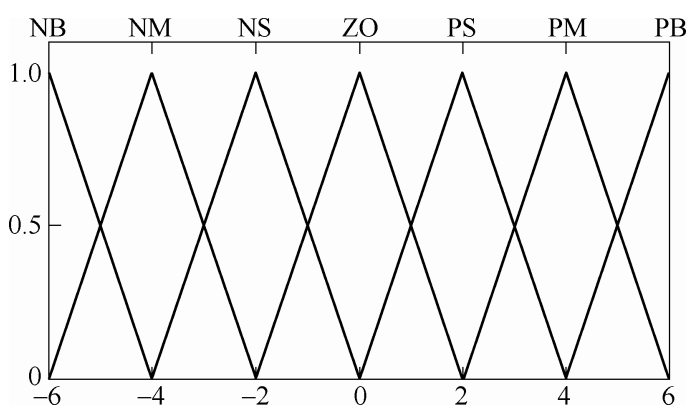

图 12 输入、输出量隶属度分布图
(3) 模糊控制规则建立。

本文中模糊控制器的输出量为 PID 参数的调整 量 $\Delta K_{\mathrm{p}}$ 和 $\Delta K_{\mathrm{i}}$, 其中, $K_{\mathrm{p}}$ 是比例调节系数, $K_{\mathrm{i}}$ 是积 分调节系数。本文根据 PID 参数对控制性能的影响 特性设计了 PID 参数调整原则, 具体如下所述。

1) 当误差 $e$ 较大时取较小的 $K_{\mathrm{i}}$ 值和较大的 $K_{\mathrm{p}}$ 值, 如此能够避免响应系统的超调量过大并快速跟 踪控制系统。

2) 当误差 $e$ 为中等大小时取适中的 $K_{\mathrm{i}}$ 值和稍 小的 $K_{\mathrm{p}}$ 值, 如此能够使系统具有具有较小的超调量 和较快的响应速度。

3) 当误差 $e$ 和误差变化率 $e_{\mathrm{c}}$ 都较小时取较大的 $K_{\mathrm{i}}$ 和 $K_{\mathrm{p}}$ 值, 而当误差 $e$ 较小但误差变化率 $e_{\mathrm{c}}$ 较大时 取较大的 $K_{\mathrm{i}}$ 和 $K_{\mathrm{p}}$ 值，如此能够使控制系统的抗干 扰能力和稳定性较高。

根据以上调整原则，参考有关文献 $[14,17]$, 本 文归纳得到 $\Delta K_{\mathrm{p}} 、 \Delta K_{\mathrm{i}}$ 的模糊控制规则分别如表 4 和表 5 所示。

表 $4 \Delta K_{\mathrm{p}}$ 的模糊控制规则表

\begin{tabular}{cccccccc}
\hline & \multicolumn{7}{c}{$e_{\mathrm{c}}$} \\
\cline { 2 - 7 } & NB & NM & NS & ZO & PS & PM & PB \\
\hline NB & PB & PB & PM & PM & PS & PS & ZO \\
NM & PB & PB & PM & PM & PS & ZO & NS \\
NS & PM & PM & PM & PS & ZO & NS & NS \\
ZO & PM & PS & PS & ZO & NS & NM & NM \\
PS & PS & PS & ZO & NS & NS & NM & NM \\
PM & PS & ZO & NS & NS & NM & NM & NM \\
PB & ZO & ZO & NM & NM & NM & NB & NB \\
\hline
\end{tabular}

表 $5 \Delta K_{\mathrm{i}}$ 的模糊控制规则表

\begin{tabular}{cccccccc}
\hline \multirow{2}{*}{$E$} & \multicolumn{7}{c}{$e_{\mathrm{c}}$} \\
\cline { 2 - 7 } & NB & NM & NS & ZO & PS & PM & PB \\
\hline NB & NB & NB & NM & NS & NS & NS & ZO \\
NM & NB & NB & NM & NM & NS & ZO & PS \\
NS & NB & NM & NS & NS & ZO & PS & PM \\
ZO & NM & NS & NS & ZO & PS & PM & PM \\
PS & NM & NS & ZO & PS & PS & PM & PB \\
PM & NS & ZO & PS & PS & PM & PB & PB \\
PB & ZO & ZO & PS & PS & PM & PB & PB \\
\hline
\end{tabular}

\section{4 基于 iFIX 组态软件的智能调控软件设计}

针对上文提到的基于 BP 神经网络的摩擦制 动力矩终值预测模型和基于模糊 PID 的复合制动 力矩反馈调控模型, 本文使用 MATLAB 软件对它 们进行了设计。对设计完成的模型的 $\mathrm{m}$ 文件进行 打包, 并生成 dll 文件, 这样可以使其脱离 MATLAB 工作环境, 而后在智能调控系统中通过 上位机软件调用该 $\mathrm{d} 11$ 文件, 即可实现摩擦制动力 矩终值预测和反馈调控复合制动力矩这两个重要 
功能。神经网络预测模型调用上位机中输入的制 动初速度和制动压力两个参数, 而后通过模糊控 制等函数调用其他参数进行相关计算, 最终得到 的励磁电流被传至上位机界面显示, 或由下位机 输出模块将信号输出至执行元件, 实现过程控制, 如图 13 所示。利用 $\mathrm{iFIX}$ 软件设计完成的智能调 控软件界面如图 14 所示。

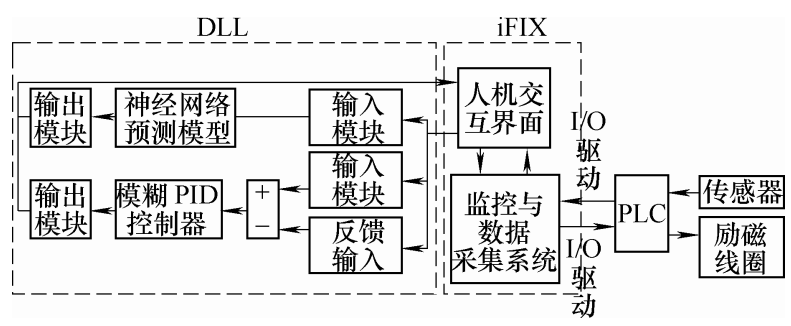

图 $13 \mathrm{iFIX}$ 与 DLL 数据交换过程示意图

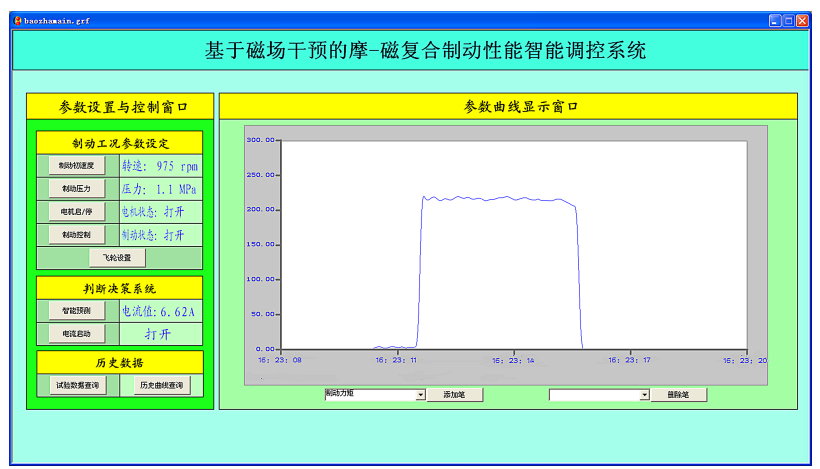

图 14 智能调控软件界面

\section{5 摩-磁复合制动恒力矩调控试验与分析}

为检验所建立的摩-磁复合制动性能智能 调控系统的工作性能, 以确定其能否通过不断 改变电磁制动力矩的方法实现制动系统恒力矩 制动, 本文在制动初速度和制动压力相同的情 况下, 分别开展常规的摩擦制动试验和摩-磁复 合制动恒力矩调控试验, 旨在通过对比的方法对 试验结果进行分析。两组试验的制动初速度均设 定为 $90 \mathrm{~km} / \mathrm{h}$ (换算成转速为 $975 \mathrm{r} / \mathrm{min}$ ), 制动压 力均设定为 $1.1 \mathrm{MPa}$, 如图 14 所示。在进行恒 力矩调控试验时, 首先在上位机输入设定好的制 动压力和制动初速度, 然后启动电动机, 点击 “智 能预测” 按钮, 判断决策系统会进行智能预测, 并显示对应的励磁电流。当电动机转速达到设定 速度时, 同时点击 “制动控制” 和 “电流启动” 按钮, 进行复合制动, 此时显示的电流值将不断 发生变化, 该变化即为反馈调节系统实时检测并 对电磁制动力矩进行调节, 直到转速为零, 以此 实现恒力矩制动。试验结果分别如图 15 和图 16 所示。

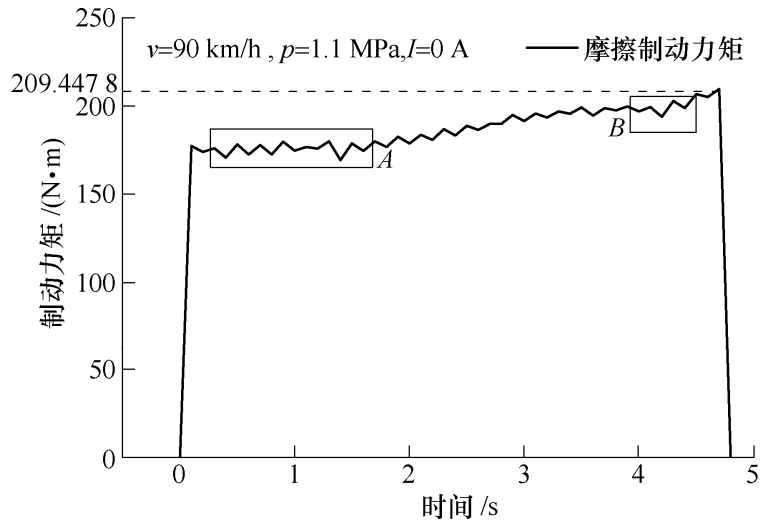

图 15 常规摩擦制动试验结果

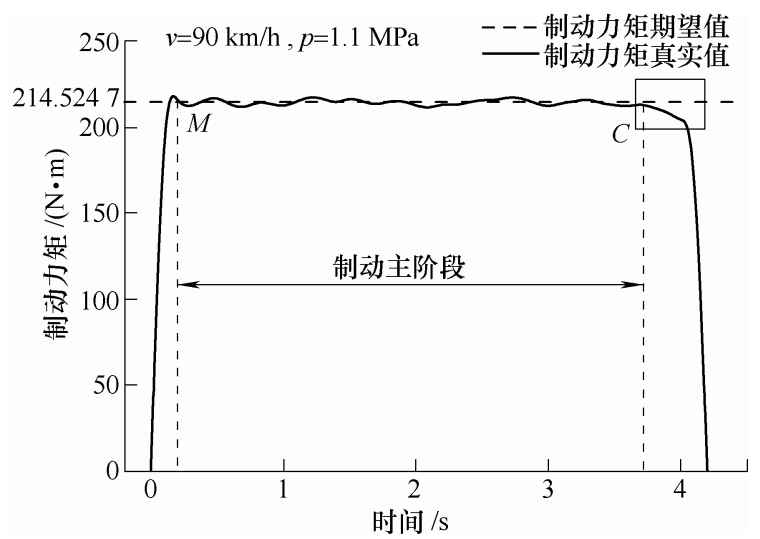

图 16 摩-磁复合制动恒力矩调控试验结果

由图 15 和图 16 可以发现以下几点。

(1) 摩擦制动力矩在常规摩擦制动试验过程中 不断波动增加, 在图 15 中通过 $A 、 B$ 标注的方框位 置处出现了较为明显的波动。

(2) 由于制动末期的速度很小, 此时电磁制动 力矩受速度的影响大于受电流的影响, 因此系统电 流的调控对其几乎不起作用; 同时根据前文分析, 磁场的出现可以降低摩擦制动力矩增加的幅度, 由 于本文在进行摩擦制动力矩终值预测模型的设计时 未考虑此影响, 因此造成预测结果的偏大。以上这 两点原因可以解释在制动末期出现的较为明显的 复合制动力矩下降现象, 如图 16 中方框标注位置 所示。

(3) 在常规摩擦制动试验中的制动力矩终值为 $209.4478 \mathrm{~N} \cdot \mathrm{m}$, 而在摩-磁复合制动恒力矩调控试验 中预测的摩擦制动力矩终值(期望值)为 214.5247 $\mathrm{N} \cdot \mathrm{m}$ (如图 16 中虚线所示), 两者的差值较小 (约为 5 $\mathrm{N} \cdot \mathrm{m}$ ), 在比较合理的范围之内。

(4) 由于复合制动力矩在制动初期的超调现象 (PID 调控造成)和制动末期的明显下降现象所持续 时间都比较短暂, 因此本文忽略两部分的影响, 将 摩-磁复合制动力矩期望值曲线与忽略超调后真实 值曲线的第一个交点(图中 $M$ 点)作为主阶段的起 点, 将复合制动力矩真实值开始出现明显下降的起 
始点(图中 $C$ 点)作为主阶段的终点, 以此构成 “制 动主阶段” 对调控性能的优劣进行评价。根据式(5) 计算得到常规摩擦制动力矩的稳定系数约为 $5.7 \%$, 而在复合制动主阶段, 摩-磁复合制动力矩的稳定系 数约为 $1.9 \%$ 。

$$
\alpha_{f}=\frac{\overline{T_{f}}-T_{f x \max }}{\overline{T_{f}}}
$$

式中, $\overline{T_{f}}$ 为复合制动力矩均值; $\alpha_{f}$ 为复合制动力 矩稳定系数; $T_{f x \text { max }}$ 为复合制动力矩的最大下降量。

\section{4 结论}

(1) 智能调控系统预测结果与常规摩擦制动试 验的制动力矩终值之差在合理范围内, 通过输入制 动压力和制动初速度两个参数, 所建立的 $\mathrm{BP}$ 神经 网络预测模型可以有效地预测摩擦制动力矩终值。

(2) 制动过程中制动力矩是动态波动变化的, 通过降低制动力矩的波动幅值, 所建立的模糊 PID 反馈调控法可以实现制动系统以近似恒力矩制动。

(3) 相较于常规的摩擦制动, 摩-磁复合制动方 式具有更大的制动力矩和更短的制动时间, 因此表 现出更好的制动性能。本文的研究结果对于提高机械 设备的制动效能与可靠性、促进多源复合制动系统调 控技术的发展具有重要的理论价值和实际意义。

\section{参 考 文 献}

[1] 张庆金, 鲍久圣, 阴妍, 等. 制动摩擦材料的研究与发 展现状[J]. 表面技术，2016，45(11): 32-40.

ZHANG Qingjin, BAO Jiusheng, YIN Yan, et al. Research and development of brake friction materials[J]. Surface Technology, 2016, 45(11): 32-40.

[2] BAO Jiusheng, LIU Jinge, YIN Yan, et al. Characterization and experiments on the friction catastrophe behaviors of brake material during emergency braking[J]. Engineering Failure Analysis, 2015，55(2): 55-62.

[3] BAO Jiusheng, CHEN Guangzhu, ZHU Zhencai, et al. Friction and wear properties of the composite brake material for mine hoister under different initial velocity[J]. Proceedings of the IMechE, Part J: Journal of Engineering Tribology, 2012, 226(10): 873-879.

[4] 王意东, 何太碧. 汽车电浴流缓速器综述 $[\mathrm{J}]$. 长江大学 学报, 2011, 8(5): 109-113.

WANG Yidong, HE Taibi. Overview of automobile eddy current retarder[J]. Journal of Yangtze University, 2011, 8(5): 109-113.
[5] CHO S, LIU Huaicong, LEE J, et al. Design and analysis of the eddy current brake with the winding change [J]. Journal of Magnetics, 2017, 22(1): 23-28.

[6] 张端军, 顾晓丹, 何仁. 电浴流制动的工作原理及其在 汽车上的应用[J]. 机械设计与制造, 2014, (1): 121-123. ZHANG Ruijun, GU Xiaodan, HE Ren. Principle and application on automobile of the eddy current brake[J]. Machinery Design \& Manufacture, 2014, (1): 121-123.

[7] 何仁, 刘存香, 李楠. 轿车电磁制动与摩擦制动集成系 统的模糊控制[J]. 机械工程学报, 2010, 46(24): 83-87. HE Ren, LIU Cunxiang, LI Nan. Fuzzy control of the integrated system of electromagnetic brake and friction brake of car[J]. Journal of Mechanical Engineering, 2010, 46(24): $83-87$.

[8] 何仁, 胡东海, 张端军. 汽车电磁制动技术的研究与进 展 $[J]$. 汽车安全与节能学报, 2013，4(3): 202-214.

HE Ren, HU Donghai, ZHANG Duanjun. Research and development of automobile electromagnetic brake technology for commercial vehicles[J]. Automotive Safety and Energy，2013，4(3): 202-214.

[9] 何仁, 刘存香. 摩擦式制动器与非接触轮边缓速器集成 系统仿真分析 [J]. 南京理工大学学报, 2011, 35(5): 590-594.

HE Ren, LIU Cunxiang. Simulation of integrated system of frictional brake and contactless wheel retarder[J]. Journal of Nanjing University of Science and Technology, 2011, 35(5): 590-594.

[10] 刘存香, 何仁. 电磁制动与摩擦制动集成系统测试台架 设计及试验研究 $[J]$. 汽车技术, 2012，(1): 51-55.

LIU Cunxiang, HE Ren. Test bench design and experimental study of integrated system of electromagnetic braking and friction braking[J]. Automobile Technology, 2012, (1): 51-55.

[11] 刘存香, 何仁, 胡春花. 轿车电磁制动与摩擦制动集成 系统的性能试验 [J]. 江苏大学学报, 2012, 33(5): 508-512.

LIU Cunxiang, HE Ren, HU Chunhua. Performance test of integrated system of car electromagnetic brake and friction brake[J]. Journal of Jiangsu University, 2012, 33(5): 508-512.

[12] 何仁, 汤宝, 赵强. 电磁与摩擦制动集成系统切换控制 策略[J]. 江苏大学学报, 2015, 36(2): 125-129.

HE Ren, TANG Bao, ZHAO Qiang. Switching control strategy of integrated electromagnetic andfriction brake system[J]. Journal of Jiangsu University, 2015，36(2): 125-129.

[13] 胡东海. 汽车摩擦制动与电磁制动的系统集成与协调 控制[D]. 镇江: 江苏大学, 2016.

HU Donghai. System integrationand coordinated control 
of frictional brake and electromagnetic brake of vehicle[J]. Zhenjiang: Jiangsu University, 2016.

[14] 纪洋洋. 基于磁场干预的摩-磁复合制动性能智能调控 方法研究[D]. 徐州: 中国矿业大学, 2017.

JI Yangyang. Intelligent controlling method of frictional-magnetic compound braking performance based on magnetic field[D]. Xuzhou: China University of Mining and Technology, 2017.

[15] 陆玉浩. 盘式制动器摩擦故障特征提取与模式识别研 究[D]. 徐州: 中国矿业大学, 2014.

LU Yuhao. Feature extraction and pattern recognition of friction faults for disc brake[D]. Xuzhou : China University of Mining and Technology, 2014.
[16] 刘金革. 汽车磁-摩复合盘式制动器及其制动性能研究 [D]. 徐州: 中国矿业大学, 2016.

LIU Jinge. Automotive magnetic-friction compound Disc brake and its braking performance[D]. Xuzhou: China University of Mining and Technology, 2016.

[17] 于秀丽, 王大志. 模糊自适应整定 PID 控制系统[J]. 沈 阳工业学院学报, 2004(2): 41-44.

YU Xiuli, WANG Dazhi. Fuzzy adaptive tuning PID control system[J]. Journal of Shenyang Institute of Technology, 2004 (2): 41-44.

作者简介: 鲍久圣(通信作者), 男, 1979 年出生, 博士, 教授, 主要研 究方向为摩擦学与制动技术。

E-mail: cumtbjs@126.com 